\title{
NUMERICAL SOLUTIONS AND THEIR SUPERCONVERGENCE FOR WEAKLY SINGULAR INTEGRAL EQUATIONS WITH DISCONTINUOUS COEFFICIENTS
}

\author{
KRISTIINA HAKK AND ARVET PEDAS \\ Institute of Applied Mathematics, University of Tartu \\ Liivi 2 - 206 , EE-2400 Tartu, Estonia \\ E-mail: arvet.pedas@ut.ee
}

\section{ABSTRACT}

The piecewise polynomial collocation method is discussed to solve second kind Fredholm integral equations with weakly singular kernels $K(t, s)$ which may be discontinuous at $s=d, d=$ const. The main result is given in Theorem 4.1. Using special collocation points, error estimates at the collocation points are derived showing a more rapid convergence than the global uniform convergence in the interval of integration available by piecewise polynomials.

\section{INTEGRAL EQUATION}

Consider the linear integral equation

$$
u(t)=\int_{0}^{b} K(t, s) u(s) d s+f(t), \quad 0 \leq t \leq b,
$$

where $b \in \mathbb{R}$ and $f:[0, b] \rightarrow \mathbb{R}$ is a given continuous function. Throughout this paper we shall suppose that the kernel $K$ has the form

$$
K(t, s)=a(t, s) \kappa(t-s)
$$

where

(A1) the function $\kappa(\tau)$ is $m-1$ times $(m \geq 1)$ continuously differentiable with respect to $\tau$ for $\tau \in[-b, b] \backslash\{0\}$ and such that the estimates

$$
\left|\kappa^{(k)}(\tau)\right| \leq b_{k}|\tau|^{-\alpha-k}, \quad k=0,1, \ldots, m-1,
$$


hold with $0<\alpha<1$ and some positive constants $b_{0}, b_{1}, \ldots, b_{m-1}$;

(A2) the function $a(t, s)$ is $m$ times continuously differentiable on $[0, b] \times[0, d]$ and $[0, b] \times[d, b]$ independently, where $d$ is a fixed point in the interval $(0, b)$.

Let $C^{m}(X)$, where $X \subset \mathbb{R}$, denote the space of $m$ times continuously differentiable functions $x: X \rightarrow \mathbb{R}$. For $0<\alpha<1, m \in \mathbb{N}, 0<d<b$, define

$$
\begin{gathered}
E^{\alpha, m} \equiv\left\{u \in C[0, b] \cap C^{m}(0, d) \cap C^{m}(d, b):\right. \\
\left.\sup _{\substack{0<t<b \\
t \neq d}} \frac{\left|u^{(m)}(t)\right|}{t^{-(\alpha+m-1)}+|t-d|^{-(\alpha+m-1)}+(b-t)^{-(\alpha+m-1)}}<\infty\right\} ;
\end{gathered}
$$

$E^{\alpha, m}$ is a Banach space under the norm

$$
\|u\|_{E^{\alpha, m}}=\max _{0 \leq t \leq b}|u(t)|+\sup _{\substack{0<t<b \\ t \neq d}} \frac{\left|u^{(m)}(t)\right|}{t^{-(\alpha+m-1)}+|t-d|^{-(\alpha+m-1)}+(b-t)^{-(\alpha+m-1)}}
$$

It follows from $u \in E^{\alpha, m}$ that $u \in C[0, b] \cap C^{m}(0, d) \cap C^{m}(d, b)$ and for $0<t<d$ and $d<t<b$ the following estimates hold:

$$
\left|u^{(k)}(t)\right| \leq c_{k}\left[t^{-(\alpha+k-1)}+|t-d|^{-(\alpha+k-1)}+(b-t)^{-(\alpha+k-1)}\right], k=1, \ldots, m,
$$

where $c_{1}, \ldots, c_{k}$ are some positive constants. Note also that $C^{m}[0, b] \subset E^{\alpha, m}$.

The following result (see $[4 ; 6 ; 2]$ ) states the regularity properties of solutions of equation (1.1).

Lemma 1.1. Let the assumptions (A1) and (A2) hold, and let $f \in E^{\alpha, m}$. If integral equation (1.1) has a solution $u \in L^{1}(0, b)$ then $u \in E^{\alpha, m}$.

REMARK 1.If the function $a(t, s)$ is continuous on $[0, b] \times[0, b]$ then the estimates (1.4) for the derivatives of the solution $u(t)$ of equation (1.1) can be specified (see [4]).

\section{PIECEWISE POLYNOMIAL APPROXIMATION}

Let $N \in \mathbb{N}, r \in \mathbb{R}, r \geq 1$. We introduce in the interval $[0, d]$ the following $2 N$ grid points

$$
\begin{aligned}
t_{j}^{(N)} & =\left(\frac{j}{N}\right)^{r} \frac{d}{2}, j=0,1, \ldots, N ; \\
t_{N+j}^{(N)} & =d-t_{N-j}^{(N)}, j=1, \ldots, N-1,
\end{aligned}
$$


and in the interval $[d, b] 2 N+1$ grid points

$$
\begin{aligned}
& t_{2 N+j}^{(N)}=d+\left(\frac{j}{N}\right)^{r} \frac{b-d}{2}, j=0,1, \ldots, N ; \\
& t_{3 N+j}^{(N)}=b-t_{3 N-j}^{(N)}, j=1, \ldots, N-1 ; \quad t_{4 N}^{(N)}=b .
\end{aligned}
$$

Here $r \geq 1$ characterizes the degree of the nonuniformity of the grid. If $r=1$ then the grid points (2.1) and (2.2) are uniformly located in the intervals $[0, d]$ and $[d, b]$ respectively (and in $[0, b]$ if $d=b / 2$ ). If $r>1$ then the grid points $\{(2.1),(2.2)\}$ are more densely located towards the end points of the intervals $[0, d]$ and $[d, b]$.

We dermine the collocation points in the following way. We choose $m$ points $\eta_{1}, \ldots, \eta_{m}$ in the interval $[-1,1]$ :

$$
-1 \leq \eta_{1}<\eta_{2}<\ldots<\eta_{m} \leq 1 \text {. }
$$

By affine transformations we transfer them into the interval $\left[t_{j-1}^{(N)}, t_{j}^{(N)}\right]$ :

$$
\xi_{j, q}^{(N)}=t_{j-1}^{(N)}+\frac{\eta_{q}+1}{2}\left(t_{j}^{(N)}-t_{j-1}^{(N)}\right), \quad q=1, \ldots, m ; j=1, \ldots, 4 N .
$$

Note that $\xi_{j, m}^{(N)}=\xi_{j+1,1}^{(N)}=t_{j}^{(N)}$, if $\eta_{1}=-1, \eta_{m}=1(j=1, \ldots, 4 N-1)$.

For a continuous function $u:[0, b] \rightarrow \mathbb{R}$ we construct a piecewise polynomial interpolation function $P_{N} u:[0, b] \rightarrow \mathbb{R}$ as follows: on every interval $\left[t_{j-1}^{(N)}, t_{j}^{(N)}\right]$ $(j=1, \ldots, 4 N), P_{N} u$ is a polynomial of degree not exceeding $m-1$ and

$$
\left(P_{N} u\right)\left(\xi_{j, q}^{(N)}\right)=u\left(\xi_{j, q}^{(N)}\right), \quad q=1, \ldots, m ; j=1, \ldots, 4 N .
$$

Thus the interpolation function $\left(P_{N} u\right)(t)$ is uniquely defined in every interval $\left[t_{j-1}^{(N)}, t_{j}^{(N)}\right](j=1, \ldots, 4 N)$ separately and may have jumps if $t=t_{j}^{(N)}$, $j=1, \ldots, 4 N-1$. If $\eta_{1}=-1, \eta_{m}=1$, then $P_{N} u$ is a continuous function on the interval $[0, b]$. We can define $\left(P_{N} u\right)(t)$ by the formula

$$
\left(P_{N} u\right)(t)=\sum_{q=1}^{m} u\left(\xi_{j, q}^{(N)}\right) \varphi_{j, q}^{(N)}(t), \quad t \in\left[t_{j-1}^{(N)}, t_{j}^{(N)}\right], j=1, \ldots, 4 N
$$

where $\varphi_{j, q}^{(N)}(t), t \in\left[t_{j-1}^{(N)}, t_{j}^{(N)}\right], q=1, \ldots, m$, are the polynomials of degree $m-1$ such that

$$
\varphi_{j, q}^{(N)}\left(\xi_{j, p}^{(N)}\right)=\left\{\begin{array}{ll}
1, & p=q \\
0, & p \neq q
\end{array}\right\}, \quad p=1, \ldots, m .
$$

Let us denote by $E_{N}$ the range of the operator $P_{N} \equiv P_{N}^{(m)}$. This is the space of piecewise polynomial functions $u_{N}$ on $[0, b]$ which on every interval $\left[t_{j-1}^{(N)}, t_{j}^{(N)}\right](j=1, \ldots, 4 N)$ are polynomials of the degree not exceeding $m-1$. 
The approximation properties of $P_{N} u$ on grid $\{(2.1),(2.2)\}$ are considered in $[5]$ (cf. also $[6 ; 7 ; 8]$ ). These results can be summarized as follows.

Lemma 2.1. Assume that $u \in E^{\alpha, m}$. Then

$$
\left\|u-P_{N} u\right\|_{L^{\infty}(0, b)} \leq \mathrm{const} \begin{cases}h_{N}^{r(1-\alpha)} & \text { for } 1 \leq r \leq \frac{m}{1-\alpha} \\ h_{N}^{m} & \text { for } r \geq \frac{m}{1-\alpha}\end{cases}
$$

where

$$
h_{N}=\max \left\{\frac{d}{2 N}, \frac{b-d}{2 N}\right\}
$$

\section{COLLOCATION METHOD}

We look for an approximate solution $u_{N} \in E_{N}$ to integral equation (1.1). We require that $u_{N}$ should satisfy the equation (1.1) at the collocation points (2.4):

$$
\begin{gathered}
{\left[u_{N}(t)-\int_{0}^{b} K(t, s) u_{N}(s) d s-f(t)\right]_{t=\xi_{i, p}^{(N)}}=0} \\
p=1, \ldots, m, \quad i=1, \ldots, 4 N .
\end{gathered}
$$

By the representation (2.5), we can find $u_{N} \in E_{N}$ in the form

$$
u_{N}(t)=\sum_{q=1}^{m} c_{j, q}^{(N)} \varphi_{j, q}^{(N)}(t), \quad t \in\left[t_{j-1}^{(N)}, t_{j}^{(N)}\right], j=1 \ldots, 4 N
$$

where, as it follows from (2.6),

$$
c_{j, q}^{(N)}=u_{N}\left(\xi_{j, q}^{(N)}\right), \quad q=1, \ldots, m ; j=1, \ldots, 4 N
$$

Now the collocation conditions (3.1) will take the following form of a system which determines the coefficients $c_{i, p}^{(N)}=u_{N}\left(\xi_{i, p}^{(N)}\right)$ :

$$
c_{i, p}^{(N)}=\sum_{j=1}^{4 N} \sum_{q=1}^{m} a_{i, p, j, q}^{(N)} c_{j, q}^{(N)}+f\left(\xi_{i, p}^{(N)}\right), \quad p=1, \ldots, m ; i=1, \ldots, 4 N
$$

where

$$
a_{i, p, j, q}^{(N)}=\int_{0}^{b} K\left(\xi_{i, p}^{(N)}, s\right) \varphi_{j, q}^{(N)}(s) d s
$$

If $\eta_{1}>-1$ or $\eta_{m}<1$, then all collocation points $\xi_{j, q}^{(N)}(q=1, \ldots, m$, $j=1, \ldots 4 N)$ are different and there are $4 m N$ collocation points. In this 
case the system (3.2) (system (3.1)) has $4 m N=\operatorname{dim} E_{N}$ equations and the same number of unknows. If $\eta_{1}=-1$ and $\eta_{m}=1$, then part of the collocation points will coincide. The number of different collocation points is $[4 N(m-1)+1]=\operatorname{dim} E_{N}$ and the system (3.2) (system (3.1)) has the same number of equations and unknows.

THEOREM 3.1. (cf. [5]). Assume that the following conditions are fulfilled: 1) the kernel (1.2) satisfies the assumptions (A1) it and (A2);2) $f \in E^{\alpha, m} ; 3$ ) the homogeneous integral equation

$$
u(t)=\int_{0}^{b} K(t, s) u(s) d s
$$

has only the trivial solution $u=0 ; 4)$ the collocation points (2.4) are used. Then the equation (1.1) has a unique solution $u^{*}$ and there exists $N_{0}$ such that, for $N \geq N_{0}$, the collocation conditions (3.1) define a unique approximation $u_{N}^{*} \in E_{N}$ to $u^{*}$. The following error estimates hold:

$$
\left\|u_{N}^{*}-u^{*}\right\|_{L^{\infty}(0, b)} \leq c \begin{cases}h_{N}^{r(1-\alpha)} & \text { for } 1 \leq r \leq \frac{m}{1-\alpha} \\ h_{N}^{m} & \text { for } r \geq \frac{m}{1-\alpha}\end{cases}
$$

where $r$ is the scaling parameter of the grid $\{(2.1),(2.2)\}, h_{N}$ is defined in (2.8) and $c$ is a positive constant independent of $h_{N}$.

Proof. We write the integral equation (1.1) in the form $u=T u+f$ where

$$
(T u)(t)=\int_{0}^{b} K(t, s) u(s) d s, \quad t \in[0, b]
$$

It follows from (A1) and (A2) (see [1]) that $T: L^{\infty}(0, b) \rightarrow C[0, b]$, moreover, $T: L^{\infty}(0, b) \rightarrow L^{\infty}(0, b)$, is compact. As the homogeneous equation $u=T u$ has only the trivial solution $u=0$, then the equation $u=T u+f$ has a unique solution $u^{*} \in L^{\infty}(0, b)$. Due to Lemma 1.1, $u^{*} \in E^{\alpha, m}$. The collocation conditions (3.1) can be written in the form

$$
u_{N}=P_{N} T u_{N}+P_{N} f
$$

where $P_{N}$ is defined in Section 2. If $N \rightarrow \infty$ then $\left\|P_{N} u-u\right\|_{L^{\infty}(0, b)} \rightarrow 0$ for every $u \in C[0, b]$. Therefore $\left\|P_{N} T-T\right\|_{L^{\infty}(0, b) \rightarrow L^{\infty}(0, b)} \rightarrow 0, N \rightarrow \infty$. From this and from the boundedness of $(I-T)^{-1}$ in $L^{\infty}(0, b)$ we obtain that $I-P_{N} T$ is invertible for sufficiently large $N \geq N_{0}$ and uniformly bounded in $N$ :

$$
\left\|\left(I-P_{N} T\right)^{-1}\right\|_{L^{\infty}(0, b) \rightarrow L^{\infty}(0, b)} \leq c .
$$


Let $N \geq N_{0}$ and $u_{N}^{*}=\left(I-P_{N} T\right)^{-1} P_{N} f$ be the solution of the equation (3.6). Then $u_{N}^{*}-u^{*}=\left(I-P_{N} T\right)^{-1}\left(P_{N} u^{*}-u^{*}\right)$ and

$$
\| u_{N}^{*}-\left.u^{*}\right|_{L^{\infty}(0, b)} \leq c|| P_{N} u^{*}-\left.u^{*}\right|_{L^{\infty}(0, b)} .
$$

Due to (2.7) we obtain the estimate (3.4).

\section{SUPERCONVERGENCE AT COLLOCATION POINTS}

Now we assume that the points (2.3) are the nodes of a quadrature formula

$$
\int_{-1}^{1} g(s) d s=\sum_{q=1}^{m} A_{q} g\left(\eta_{q}\right)+R_{m}(g), \quad-1 \leq \eta_{1}<\ldots<\eta_{m} \leq 1,
$$

which is exact for all polynomials of degree $m+1(m \geq 2)$.

Theorem 4.1. Let $m \in \mathbb{N}, m \geq 2$. Assume that the following conditions are fulfilled:

(i) the kernel $K$ has the form (1.2) where

1) the function $\kappa(\tau)$ is $m$ times continuously differentiable with respect to $\tau$ for $\tau \in[-b, b] \backslash\{0\}$ and such that the estimates

$$
\left|\kappa^{(k)}(\tau)\right| \leq b_{k}|\tau|^{-\alpha-k}, \quad k=0,1, \ldots, m,
$$

hold with $0<\alpha<1$ and some positive constants $b_{0}, b_{1}, \ldots, b_{m}$;

2) the function $a(t, s)$ is $m+1$ times continuously differentiable on $[0, b] \times$ $[0, d]$ and $[0, b] \times[d, b]$, where $0<d<b$;

(ii) $f \in E^{\alpha, m+1}$;

(iii) homogeneous integral equation (3.3) has only the trivial solution $u=0$;

(iv) the grid $\{(2.1),(2.2)\}$ is used with $r \geq(m+1) /(1-\alpha)$ and the collocation points (2.4) are generated by the nodes (2.3) of a quadrature formula (4.1) which is exact for all polynomials of degree $m+1$.

Then there exists $N_{0} \in \mathbb{N}$ such that for $N \geq N_{0}$

$$
\max _{q=1, \ldots, m ; j=1, \ldots, 4 N}\left|u_{N}^{*}\left(\xi_{j, q}^{(N)}\right)-u^{*}\left(\xi_{j, q}^{(N)}\right)\right| \leq c h_{N}^{m} h_{N}^{1-\alpha},
$$

where $u^{*}$ is the solution of equation (1.1), $u_{N}^{*} \in E_{N}$ is the solution of the system (3.1), $h_{N}$ is defined in (2.8) and $c$ is a positive constant independent of $h_{N}($ of $N)$. 
Proof. Due to Lemma 1.1, $u^{*} \in E^{\alpha, m+1}$. We have $\left|u_{N}^{*}\left(\xi_{j, q}^{(N)}\right)-u^{*}\left(\xi_{j, q}^{(N)}\right)\right| \leq \| u_{N}^{*}-\left.P_{N} u^{*}\right|_{L^{\infty}(0, b)} \quad(q=1, \ldots, m ; j=1, \ldots, 4 N)$.

As

$$
u_{N}^{*}-P_{N} u^{*}=\left(I-P_{N} T\right)^{-1} P_{N} T\left(P_{N} u^{*}-u^{*}\right) \quad N \geq N_{0},
$$

then with help of (3.7)

$$
\left\|u_{N}^{*}-P_{N} u^{*}\right\|_{L^{\infty}(0, b)} \leq c\left\|T\left(P_{N} u^{*}-u^{*}\right)\right\|_{L^{\infty}(0, b)} .
$$

Let us estimate $\left\|T\left(P_{N} u^{*}-u^{*}\right)\right\|_{L^{\infty}(0, b)}$. Fix $t \in[0, b]$ and let

$$
\eta\left(t, h_{N}\right)=\left(t-h_{N}, t+h_{N}\right) \cap[0, b] .
$$

Then

$$
\left|\int_{0}^{b} K(t, s)\left[u^{*}(s)-\left(P_{N} u^{*}\right)(s)\right] d s\right| \leq I_{1}(t)+I_{2}(t)
$$

where

$$
\begin{aligned}
& I_{1}(t)=\sum_{j:\left[t_{j-1}^{(N)}, t_{j}^{(N)}\right] \cap \eta\left(t, h_{N}\right) \neq 0} \int_{t_{j-1}^{(N)}}^{t_{j}^{(N)}}\left|K(t, s)\left[u^{*}(s)-\left(P_{N} u^{*}\right)(s)\right]\right| d s, \\
& I_{2}(t)=\sum_{j:\left[t_{j-1}^{(N)}, t_{j}^{(N)}\right] \cap \eta\left(t, h_{N}\right)=0} \int_{t_{j-1}^{(N)}}^{t_{j}^{(N)}}\left|K(t, s)\left[u^{*}(s)-\left(P_{N} u^{*}\right)(s)\right]\right| d s .
\end{aligned}
$$

It follows from the assumption (i) that

$$
I_{1}(t) \leq c|| u^{*}-P_{N} u^{*} \|_{L^{\infty}(0, b)} \sum_{j:\left[t_{j-1}^{(N)}, t_{j}^{(N)}\right] \cap \eta\left(t, h_{N}\right) \neq 0} \int_{t_{j-1}^{(N)}}^{t_{j}^{(N)}}|t-s|^{-\alpha} d s .
$$

By the Lemma 2.1 we obtain $\left\|u^{*}-P_{N} u^{*}\right\|_{L^{\infty}(0, b)} \leq c^{\prime} h_{N}^{m}$. Due to (4.5)

$$
\sum_{j:\left[t_{j-1}^{(N)}, t_{j}^{(N)}\right] \cap \eta\left(t, h_{N}\right) \neq 0} \int_{t_{j-1}^{(N)}}^{t_{j}^{(N)}}|t-s|^{-\alpha} d s \leq c^{\prime \prime} \int_{t-2 h_{N}}^{t+2 h_{N}}|t-s|^{-\alpha} d s \leq c^{\prime \prime \prime} h_{N}^{m+1-\alpha} .
$$


Thus

$$
I_{1}(t) \leq c_{1} h_{N}^{m} h_{N}^{1-\alpha}, \quad t \in[0, b], \quad c_{1}=\text { const } .
$$

Consider the term $I_{2}(t), t \in[0, b]$. Let

$$
t_{j, \frac{1}{2}}^{(N)}=\frac{t_{j-1}^{(N)}+t_{j}^{(N)}}{2} .
$$

In addition to the points $(2.3)$ we fix in $[-1,1]$ a point $\eta_{m+1}\left(\eta_{m+1} \neq \eta_{i}\right.$, $i=1, \ldots, m)$. By an affine transformation we transfer $\eta_{m+1}$ into the point $\xi_{j, m+1}^{(N)} \in\left[t_{j-1}^{(N)}, t_{j}^{(N)}\right]$ so that $\xi_{j, m+1}^{(N)} \neq \xi_{j, i}^{(N)}, i=1, \ldots, m(j=1, \ldots 4 N)$. Similarly to the definition of $P_{N}$ (see Section 2) we define for a continuous function $u:[0, b] \rightarrow \mathbb{R}$ a piecewise polynomial function $P_{N}^{(m+1)} u:[0, b] \rightarrow \mathbb{R}$ as follows: $P_{N}^{m+1} u$ is on every interval $\left[t_{j-1}^{(N)}, t_{j}^{(N)}\right](j=1, \ldots, 4 N)$ a polynomial of degree not exceeding $m$ and

$$
P_{N}^{(m+1)} u\left(\xi_{j, q}^{(N)}\right)=u\left(\xi_{j, q}^{(N)}\right), \quad q=1, \ldots, m+1 ; j=1, \ldots, 4 N
$$

We have

$$
I_{2}(t) \leq I_{21}(t)+I_{22}(t)+I_{23}(t), \quad t \in[0, b],
$$

where

$$
\begin{aligned}
& I_{21}(t)=\sum_{j:\left[t_{j-1}^{(N)}, t_{j}^{(N)}\right] \cap \eta\left(t, h_{N}\right)=\emptyset} \int_{t_{j-1}^{(N)}}^{t_{j}^{(N)}}\left|K(t, s)-K\left(t, t_{j, \frac{1}{2}}^{(N)}\right)\right|\left|u^{*}(s)-\left(P_{N} u^{*}\right)(s)\right| d s, \\
& I_{22}(t)=\sum_{j:\left[t_{j-1}^{(N)}, t_{j}^{(N)}\right] \cap \eta\left(t, h_{N}\right)=\emptyset}\left|K\left(t, t_{j, \frac{1}{2}}^{(N)}\right)\right| \int_{t_{j-1}^{(N)}}^{t_{j}^{(N)}}\left|u^{*}(s)-\left(P_{N} u^{*}\right)(s)\right| d s, \\
& I_{23}(t)=\left|\sum_{j:\left[t_{j-1}^{(N)}, t_{j}^{(N)}\right] \cap \eta\left(t, h_{N}\right)=\emptyset} \int_{t_{j-1}^{(N)}}^{(N)} K\left(t, t_{j, \frac{1}{2}}^{(N)}\right)\left[\left(P_{N}^{(m+1)} u^{*}\right)(s)-\left(P_{N} u^{*}\right)(s)\right] d s\right| .
\end{aligned}
$$

Let us consider $I_{21}(t), t \in[0, b]$. It follows from Lemma 2.1 that

$$
I_{21}(t) \leq c h_{N}^{m} \sum_{j:\left[t_{j-1}^{(N)}, t_{j}^{(N)}\right] \cap \eta\left(t, h_{N}\right)=\emptyset} \int_{t_{j-1}^{(N)}}^{t_{j}^{(N)}}\left|\frac{\partial K(t, s)}{\partial s}\right|_{s=\tau_{j}}\left|s-t_{j, \frac{1}{2}}^{(N)}\right| d s,
$$

where $\tau_{j} \in\left(s, t_{j, \frac{1}{2}}^{(N)}\right)$. We have for $s \in\left[t_{j-1}^{(N)}, t_{j}^{(N)}\right]$

$$
\left|\frac{\partial K(t, s)}{\partial s}\right|_{s=\tau_{j}}\left|s-t_{j, \frac{1}{2}}^{(N)}\right| \leq c^{\prime} h_{N}\left|t-\tau_{j}\right|^{-\alpha-1} .
$$


Since $\left[t_{j-1}^{(N)}, t_{j}^{(N)}\right] \cap \eta\left(t, h_{N}\right)=\emptyset, s \in\left[t_{j-1}^{(N)}, t_{j}^{(N)}\right]$ and $\tau_{j} \in\left(s, t_{j, \frac{1}{2}}^{(N)}\right)$, then

$$
\tilde{c}_{1} \leq \frac{\left|t-\tau_{j}\right|}{|t-s|} \leq \tilde{c}_{2}
$$

where $\tilde{c}_{1}$ and $\tilde{c}_{2}$ are some positive constants. Therefore

$$
\begin{gathered}
I_{21}(t) \leq c^{\prime \prime} h_{N}^{m+1} \sum_{j:\left[t_{j-1}^{(N)}, t_{j}^{(N)}\right] \cap \eta\left(t, h_{N}\right)=\emptyset} \int_{t_{j-1}^{(N)}}^{t_{j}^{(N)}}|t-s|^{-\alpha-1} d s \leq \\
\leq c^{\prime \prime \prime} h_{N}^{m+1} \int_{[0, b] \backslash \eta\left(t, h_{N}\right)}|t-s|^{-\alpha-1} d s .
\end{gathered}
$$

Due to (4.5) $\int_{[0, b] \backslash \eta\left(t, h_{N}\right)}|t-s|^{-\alpha-1} d s \leq c^{\prime \prime \prime \prime} h_{N}^{-\alpha}$. Thus

$$
I_{21}(t) \leq c_{2} h_{N}^{m} h_{N}^{1-\alpha}, \quad t \in[0, b], c_{2}=\text { const } .
$$

Let us turn to $I_{22}(t), t \in[0, b]$. It follows from $\left[t_{j-1}^{(N)}, t_{j}^{(N)}\right] \cap$ $\eta\left(t, h_{N}\right)=\emptyset$ that $\left|K\left(t, t_{j, \frac{1}{2}}^{(N)}\right)\right| \leq c\left|t-t_{j, \frac{1}{2}}^{(N)}\right|^{-\alpha} \leq c^{\prime} h_{N}^{-\alpha}$. Due to Lemma 2.1 $\| u^{*}-\left.P_{N}^{(m+1)} u^{*}\right|_{L^{\infty}(0, b)} \leq c^{\prime \prime} h_{N}^{m+1}$. Therefore

$$
I_{22}(t) \leq c_{3} h_{N}^{m} h_{N}^{1-\alpha}, \quad t \in[0, b], c_{3}=\text { const. }
$$

Consider $I_{23}(t), t \in[0, b]$. Due to the assumption $(i v)$ we obtain that the quadrature formula

$$
\int_{t_{j-1}^{(N)}}^{t_{j}^{(N)}} g(s) d s=\frac{t_{j}^{(N)}-t_{j-1}^{(N)}}{2} \sum_{q=1}^{m} A_{q} g\left(\xi_{j, q}^{(N)}\right)+\frac{t_{j}^{(N)}-t_{j-1}^{(N)}}{2} R_{m}(g)
$$

remains to be exact for polynomials of degree $m+1$. Using this we have

$$
\int_{t_{j-1}}^{t_{j}}\left[\left(P_{N}^{(m+1)} u^{*}\right)(s)-\left(P_{N} u^{*}\right)(s)\right] d s=0,
$$

and therefore $I_{23}(t)=0, t \in[0, b]$. Thus

$$
I_{2}(t) \leq c_{4} h_{N}^{m} h_{N}^{1-\alpha}, \quad t \in[0, b], c_{4}=\text { const. }
$$


Now the estimate (4.2) follows from (4.3), (4.4), (4.6), (4.7) and (4.8).

REmark 2. For $a \in C^{m+1}([0, b] \times[0, b])$ the estimate (4.2) follows from the corresponding results in $[6 ; 3]$.

\section{REFERENCES}

[1] Kantorovitch L.V., Akilov G.P. Functional Analysis. Nauka, Moscow, 1977 (in Russian).

[2] Pedas A., Vainikko G. The smoothness of solutions to nonlinear weakly singular equations. Z. Anal. Anwendungen, 13 (1994), 463-467.

[3] Pedas A., Vainikko G. Superconvergence of piecewise polynomial collocations for nonlinear weakly singular integral equations. J. Integral Equations Appl., 9 (1997), 4, 380-406.

[4] Uba P. Smoothness of the solution of the weakly singular integral equation with discontinuous coefficient (in Russian). Proc. Estonian Acad. Sci. Phys. Math., 37 (1988), 2, 192-203.

[5] Uba P. Approximate solution of the weakly singular integral equation with a discontinuous coefficient (in Russian). Acta et Comment. Univ. Tartuensis, 833 (1988), 28-34.

[6] Vainikko G. Multidimensional weakly singular integral equations. Springer Verlag, Berlin, 1993.

[7] Vainikko G., Pedas A., Uba P. Methods for solving weakly singular integral equations. Tartu, 1984 (in Russian).

[8] Vainikko G, Uba P. A piecewise polynomial approximation to the solution of an integral equation with weakly singular kernel. I. Austral. Math. Soc., 22 (1981), 431-438. 